\title{
Synthesis, docking study and biological evaluation of some new thiourea derivatives bearing benzenesulfonamide moiety
}

\author{
Mostafa M. Ghorab ${ }^{1,2^{*}}$ (D, Mohamed S. A. El-Gaby ${ }^{3}$, Aiten M. Soliman², Mansour S. Alsaid ${ }^{1}$, Marwa M. Abdel-Aziz ${ }^{4}$ \\ and Mahmoud M. Elaasser ${ }^{4}$
}

\begin{abstract}
Background: A series of novel N-(2, 6-dimethoxypyrimidin-4-yl)-4-(3-(aryl)thioureido) benzenesulfonamides 3a-t was synthesized by the addition of $\mathrm{N}$-(2,6-dimethoxypyrimidin-4-yl)-4-isothiocyanatobenzenesulfonamide $\mathbf{2}$ to the appropriate aromatic amine. The structures of the synthesized compounds were inspired from the second line antituberculosis pro-drugs.

Results: Most of the new compounds were screened for their activity against Mycobacterium tuberculosis. The results of the antimycobacterial assay showed that compound $\mathbf{3 i}$ exerted the highest activity ( $\mathrm{MIC}=3.13 \mu \mathrm{g} / \mathrm{mL}$ ), followed by compound $\mathbf{3 s}$ ( $\mathrm{MIC}=6.25 \mu \mathrm{g} / \mathrm{mL}$ ).

Conclusion: The structure-activity relationship (SAR) analysis revealed that the introduction of the benzo[1,3]dioxol moiety in $\mathbf{3 i}$ and the 4-morpholinyl-4-phenyl moiety in $\mathbf{3 s}$ has proven to give the most potent compounds in this study. Docking of the promising compounds inside the active site of $M$. tuberculosis enoyl reductase InhA was performed in order to emphasize the results. The compounds showed a similar orientation to that of GSK 625 inside the active site of $\mathbf{5 J F O}$ and bind to Met 98 in a way similar to that of the co-crystallized ligand.
\end{abstract}

Keywords: Thiourea, Sulfonamides, Structure-activity relationship, Antimycobacterial

\section{Background}

Tuberculosis (TB), is a disease caused by the facultative intracellular bacterium called Mycobacterium tuberculosis (MTB). WHO declared TB as a global health crisis [1] and a main cause of death due to the lack of appropriate treatment against resistant strains [2]. In 2012, TB was responsible for the death of 1.3 million people worldwide, Over $95 \%$ of them were from developing countries, also, TB represents the third cause of death for women aged 15-44. In addition, about one-third of the world's population harbors a dormant MTB infection, representing a significant incidence of the disease for the future [3]. TB treatment is tedious and time-consuming, that requires direct therapy and follow-up for not less than 6 months

\footnotetext{
*Correspondence: mmsghorab@yahoo.com; mghorab@ksu.edu.sa 1 Department of Pharmacognosy, College of Pharmacy, King Saud University, P.O. Box 2457, Riyadh 11451, Saudi Arabia

Full list of author information is available at the end of the article
}

using these four drugs (isoniazid, rifampicin, pyrazinamide and ethambutol $[1,4]$. In addition, the recurrences of latent tuberculosis, are particularly prevalent in individuals with compromised immune system [5]. However, the present treatment protocols have proven to be underwhelming due to drug-drug interactions, intolerance, drug toxicity and poor patient adherence due to the lengthy treatment protocols $[1,6]$. That's why more effective and shorter treatment regimens are required.

Thioureas act as precursors for the synthesis of different classes of acyclic and heterocyclic compounds, in addition to their high biological activity [7-10]. Second line antituberculosis pro-drugs as thioacetazone which is useful in preventing resistance to more powerful drugs such as isoniazid, isoxyl (thiocarlide) that is effective against multi-drug resistant strains, ethionamide (ETH) and prothionamide (Fig. 1) [11-17], were used to inspire the structures of our new thiourea derivatives, together 
<smiles>CC(=O)Nc1ccc(/C=N/NC(N)=S)cc1</smiles>

Thiacetazone<smiles>CCc1cc(C(N)=S)ccn1</smiles>

Ethionamide (ETH)<smiles>CC(C)CCOc1ccc(NC(=S)Nc2ccc(OCCC(C)C)cc2)cc1</smiles>

Isoxyl (thiocarlide)

Fig. 1 Second line antituberculosis pro-drugs<smiles>CCCc1cc(C(N)=S)ccn1</smiles>

Prothionamide with their mode of action. On the other hand, sulfonamides were largely employed as preventive and chemotherapeutic agents against various diseases [18], recent studies have shown that sulfonamides also possess antimycobacterial activity [19].

For the above-mentioned reasons and as a part of our interest in the synthesis and screening of potentially bioactive compounds [20-24], we herein, report the synthesis of some novel $\mathrm{N}$-(2,6-dimethoxypyrimidin4-yl)-4-(3-(aryl)thioureido)benzenesulfonamides 3a-t to be evaluated for their antimycobacterial activity. The promising compounds $3 \mathbf{i}$ and $3 \mathbf{s}$ were docked inside the active site of $M$. tuberculosis enoyl reductase InhA, to predict their possible mode of action. InhA enzyme was chosen as it contains a very hydrophobic site that favorably interacts with thioamide or thiourea moieties [25].

\section{Results and discussion \\ Chemistry}

Isothiocyanates are widely used building blocks in the synthesis of nitrogen, sulfur and oxygen heterocycles [26]. The high electrophilicity and nucleophilicity associated with the carbon and sulfur atoms, respectively, of the isothiocyanates and their extended $\pi$ electron system make them unique precursors for a large variety of target molecules. The intermediate, $N$-(2,6-dimethoxypyrimidin-4-yl)-4-isothiocyanatobenzenesulfonamide 2 [27] used for the preparation of the target compounds have been synthesized via thiophosgenation of sulfadimethoxine $\mathbf{1}$ at room temperature in the presence of dilute hydrochloric acid, according to the reported procedure (Scheme 1).

A series of $N$-(2,6-dimethoxypyrimidin-4-yl)-4-(3-(aryl) thioureido) benzenesulfonamides $\mathbf{3 a}-\mathbf{t}$ was prepared by condensation of aromatic amines with $\mathrm{N}$-(2,6-dimethoxypyrimidin-4-yl)-4-isothiocyanatobenzenesulfonamide 2 [27] in dioxane at reflux temperature in the presence of catalytic amounts of triethylamine, (Scheme 1). The structures of synthesized compounds $\mathbf{3 a}-\mathbf{t}$ were confirmed by the absence of characteristic absorption band at $2000-2200 / \mathrm{cm}(\mathrm{N}=\mathrm{C}=\mathrm{S})$. Also, the IR of 3 is characterized by the presence of $\mathrm{NH}$, thiocarbonyl $(\mathrm{C}=\mathrm{S})$ and $\mathrm{SO}_{2}$ absorption bands. For example, the ${ }^{1} \mathrm{H}$ NMR spectrum of compound $\mathbf{3 b}$ showed two singlets at $\delta 3.81$ and $3.84 \mathrm{ppm}$ which were assigned for the two methoxy protons, a singlet at $\delta 6.1 \mathrm{ppm}$ assigned to the pyrimidine- $\mathrm{H}$, two downfield shifted singlets at $\delta 11.5$ and $11.9 \mathrm{ppm}$ which were readily assigned to the $\mathrm{HN}(1)$ and $\mathrm{HN}(2)$ protons, in addition to the presence of methyl, $\mathrm{SO}_{2} \mathrm{NH}$ and aromatic protons. The thiocarbonyl group of the thiourea moiety was also observed in the ${ }^{13} \mathrm{C}$-NMR spectrum. The formation of thioureas $3 \mathbf{a}-\mathbf{t}$ can be explained through the previously reported mechanism [24].

\section{In vitro antimycobacterial activity evaluation}

Evaluation of the synthesized compounds against $M$. tuberculosis (RCMB 010126) was initially carried out using the microplate Alamar blue assay (MABA) at the Regional Center for Mycology and Biotechnology (RCMB), Al-Azhar University (Cairo, Egypt) at a concentration of $200 \mu \mathrm{g} / \mathrm{mL}$ (Table 1). As seen in Table 1, compound $3 \mathbf{i}$ was the most potent analog exhibiting good antimycobacterial activity that produced growth inhibition of $74.9 \%$.

The results of the antimycobacterial activity as minimum inhibitory concentration (MIC) are presented in Table 2 and confirming that compound $3 \mathbf{i}$ exerted the highest antimycobacterial activity $(\mathrm{MIC}=3.13 \mu \mathrm{g} /$ 


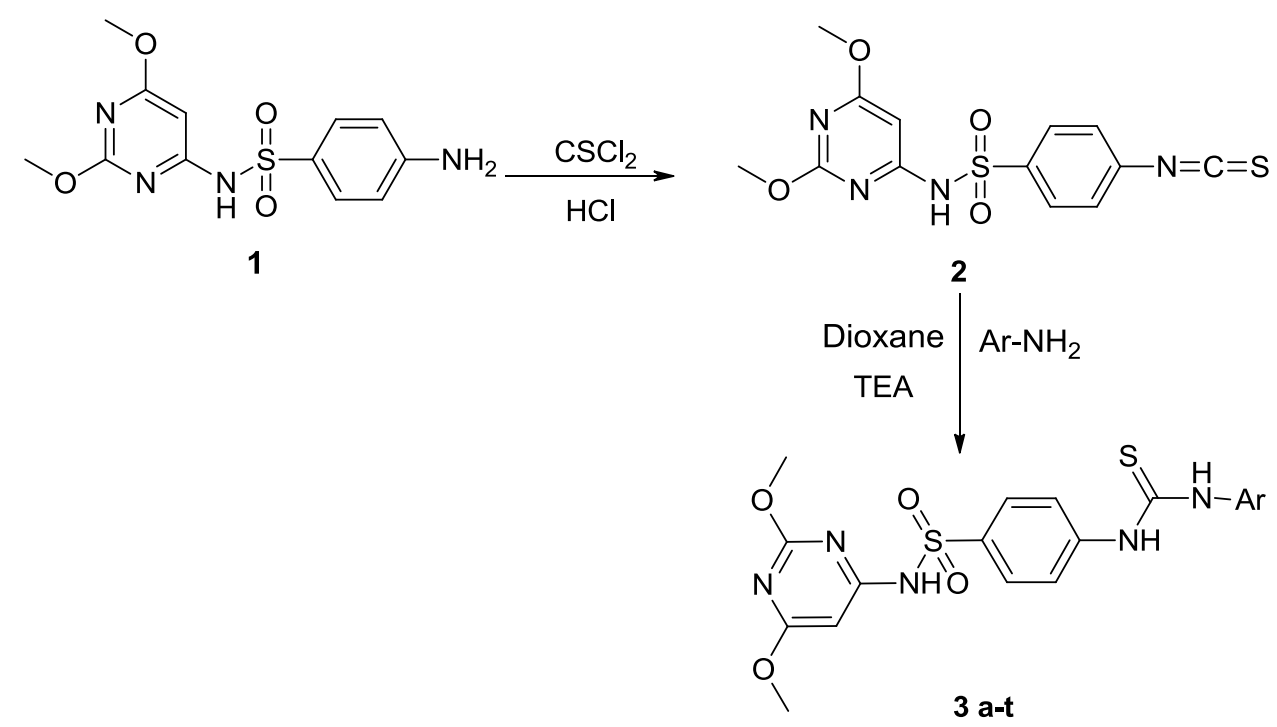<smiles></smiles><smiles></smiles><smiles>COc1cc(C)cc(OC)c1</smiles><smiles>COc1cc(C=[14CH][AsH2])cc(OC)c1OC</smiles><smiles></smiles>

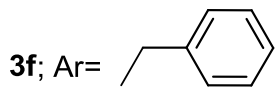<smiles>COc1cccc(CC=[Al][As])c1OC</smiles>
3h; $\mathrm{Ar}=$<smiles>CCCc1ccccc1</smiles><smiles>[Al]=[Al]Cc1ccc2c(c1)OCO2</smiles><smiles>CCc1ccc2c(c1)OCO2</smiles><smiles></smiles><smiles>Cc1cccc2c1CCCC2</smiles><smiles>Cc1ccc2c(c1)CCC2</smiles>

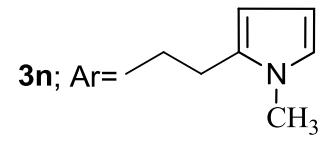<smiles></smiles><smiles>CN1C(=O)c2ccc([Te][As])cc2C1=O</smiles><smiles>[As]=[As]c1ccc(N2CCOCC2)cc1</smiles>
3t; $\mathrm{Ar}=$<smiles>CC12C=C3CC(CC(C3)C1)C2</smiles><smiles></smiles><smiles>[17C][Mg]Cn1c(-c2ccccn2)nnc1-c1ccccn1</smiles> 
Table 1 The inhibitory activities of the synthesized compounds against Mycobacterium tuberculosis

\begin{tabular}{lll}
\hline Sample code & \% Inhibition & SD \\
\hline $\mathbf{3 a}$ & 25.3 & 1.1 \\
$\mathbf{3 b}$ & 0 & 0 \\
$\mathbf{3 c}$ & 0 & 0 \\
$\mathbf{3 d}$ & 8.9 & 0.3 \\
$\mathbf{3 e}$ & 36.2 & 2.1 \\
$\mathbf{3 f}$ & 11.3 & 0.8 \\
$\mathbf{3 g}$ & 14.7 & 0.6 \\
$\mathbf{3 i}$ & 74.9 & 4.3 \\
$\mathbf{3 1}$ & 12.5 & 1.1 \\
$\mathbf{3 n}$ & 0 & 0 \\
$\mathbf{3 0}$ & 0 & 0 \\
$\mathbf{3 p}$ & 23.9 & 1.4 \\
$\mathbf{3 q}$ & 41.2 & 2.8 \\
$\mathbf{3 r}$ & 53.8 & 2.6 \\
$\mathbf{3 s}$ & 59.2 & 4.3 \\
$\mathbf{3 t}$ & 10.3 & 0.8 \\
Isoniazid & 93.5 & 1.4 \\
\hline
\end{tabular}

$\mathrm{mL}$ ), followed by compound $3 \mathrm{~s}$ (MIC $=6.25 \mu \mathrm{g} /$ $\mathrm{mL}$ ) then compounds 3r, 3q, 3e, 3a, 3p, 3g, 3l, 3f, 3t and 3d, respectively. On the other hand, compounds $\mathbf{3 b}, \mathbf{3 c}, \mathbf{3 n}$ and $3 \mathbf{o}$ exhibited no antimycobacterial activity under these experimental conditions.

From the results in Table 2, it is apparent that the 4-position of the thiourea derivatives $3 \mathbf{a}-\mathbf{t}$, crucially affected the antimycobacterial activity. In which, incorporation of a Benzo[1,3]dioxol group in compound $3 \mathbf{i}$ led to good activity against $M$. tuberculosis $(\mathrm{MIC}=3.13 \mu \mathrm{g} / \mathrm{mL})$. The introduction of a methoxy group at 2-position of the spirodecane system increased the activity (except for $\mathbf{3 b}$ ). The introduction of an electron-donating group at the 4-position, as methyl and methoxy groups, increased the activity. However, di- and trimethoxy substitutions (compounds 3c, 3d and 3g) led to decrease in the lipophilicity with a subsequent decrease in the antimycobacterial activity, indicating that the increased lipophilicity is crucial for the antitubercular activity.

It is well documented that increasing the lipophilicity, increases the diffusion through the lipid domain, thus, increasing the efficacy of the antimycobacterial agent [28-31].

\section{Molecular docking}

Tuberculosis is characterized by a number of drug targets namely: InhA, RpoB, DNA Gyrase, ATP synthase, and DprE1, inhibitors of those targets were found to be promising leads [32]. Isoniazid is still the most potent
Table 2 The estimated minimum inhibitory concentrations (MICs) of the synthesized compounds against Mycobacterium tuberculosis

\begin{tabular}{lll}
\hline Tested compounds & MIC values $(\boldsymbol{\mu g} / \mathbf{m L})$ & MIC $(\boldsymbol{\mu M})$ \\
\hline $\mathbf{3 a}$ & 50 & 98.8 \\
$\mathbf{3 b}$ & $\mathrm{NA}$ & $\mathrm{NA}$ \\
$\mathbf{3 c}$ & $\mathrm{NA}$ & $\mathrm{NA}$ \\
$\mathbf{3 d}$ & 200 & 373.8 \\
$\mathbf{3 e}$ & 50 & 102.2 \\
$\mathbf{3 f}$ & 200 & 435.7 \\
$\mathbf{3 g}$ & 100 & 192.7 \\
$\mathbf{3 i}$ & 3.13 & 6.4 \\
$\mathbf{3 I}$ & 200 & 400.8 \\
$\mathbf{3 n}$ & $\mathrm{NA}$ & $\mathrm{NA}$ \\
$\mathbf{3 0}$ & $\mathrm{NA}$ & $\mathrm{NA}$ \\
$\mathbf{3 p}$ & 100 & 173.9 \\
$\mathbf{3 q}$ & 25 & 43.5 \\
$\mathbf{3 r}$ & 12.5 & 21.7 \\
$\mathbf{3 s}$ & 6.25 & 11.8 \\
$\mathbf{3 t}$ & 200 & 397.6 \\
Isoniazid & 0.195 & 1.42 \\
\hline
\end{tabular}

$N A$ no anti-TB activity under the screening conditions

treatment targeting InhA enzyme. Isoniazid was found to interfere with Nicotinamide adenine dinucleotide (NAD)-utilizing enzymes, primarily the enoyl-ACP reductase encoded by the InhA gene, leading to the arrest of mycolic acid synthesis, which is essential to M. tuberculosis [32, 33]. InhA enzyme was chosen based upon its hydrophobic properties that favorably interact with thioamide or thiourea moieties [25].

In our present study to determine the possible mode of action of the target compounds, molecular docking of compounds $3 \mathbf{i}$ and $3 \mathbf{s}$ was performed in the active site of Mycobacterium tuberculosis enoyl reductase InhA to explore their possible binding modes. The protein data bank file (PDB: 5JFO) was selected for this purpose. The file contains $M$. tuberculosis enoyl reductase InhA enzyme co-crystallized with $N$-[1-[(2-chloro-6-fluorophenyl)methyl]-1H-pyrazol-3yl]-5-[(1S)-1-(3-methyl-1H-pyrazol- 1-yl)ethyl]-1,3,4thiadiazol-2-amine (GSK 625) [34]. All docking procedures were carried out using molecular operating environment (MOE) software 10.2008. Docking protocol was verified by re-docking of the co-crystallized ligand in the binding pocket of the enzyme with energy score $(S)=-10.44 \mathrm{kcal} / \mathrm{mol}$ and root mean standard deviation $($ RMSD) $=0.39$ (Fig. 2). The 2D ligand interaction of compound 3i (Fig. 3) demonstrates that the compound binds to the amino acid of the active site Met 98 through two hydrogen bonds (1.72, $2.44 \AA$ ). 


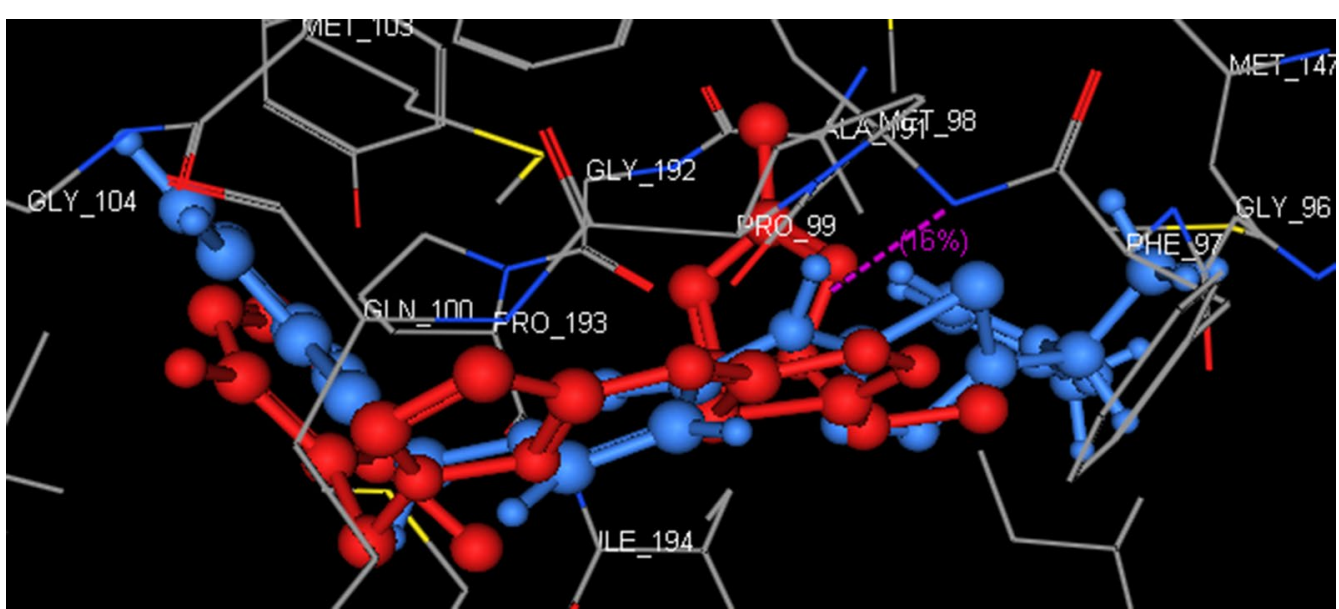

Fig. 2 Superimposition of the co-crystallized ligand (red) and the re-docked ligand (b/ue) inside the active site of $\mathbf{5}$ JFO

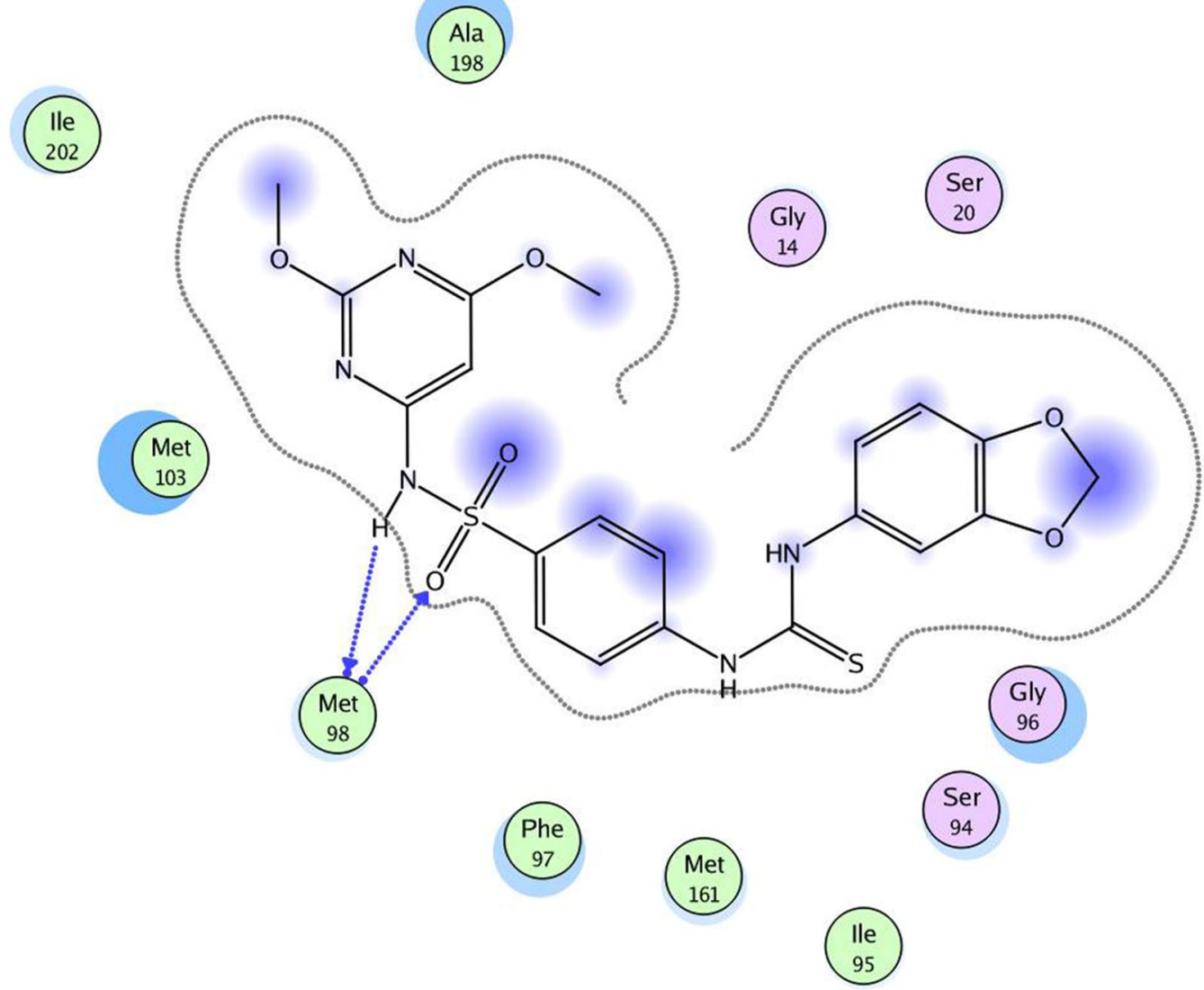

Fig. $32 D$ interactions of compound $\mathbf{3 i}$ with the active site amino acids of $\mathbf{5} \mathbf{J F O}$

Regarding compound 3s, the 2D and 3D ligand interaction simulations (Figs. 4 and 5) showed that $3 \mathbf{s}$ binds in the same fashion to the co-crystallized ligand displaying two hydrogen bonds with the active pocket amino acid Met 98 leading to an overall binding energy of $=-11.64 \mathrm{kcal} / \mathrm{mol}$ (Table 3$)$. 


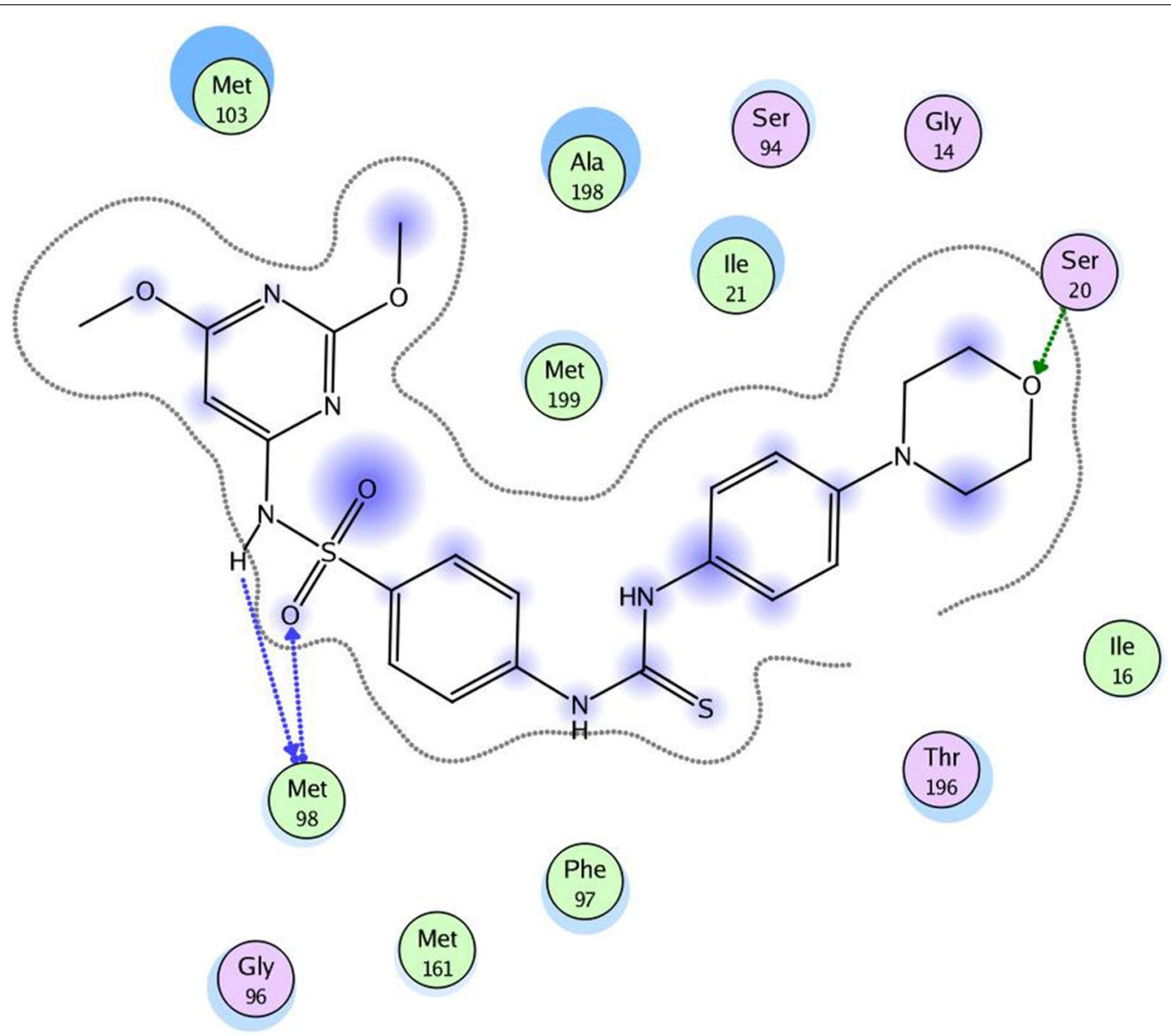

Fig. $42 \mathrm{D}$ interactions of compound $\mathbf{3 s}$ with the active site amino acids of $\mathbf{5} \mathbf{J F O}$

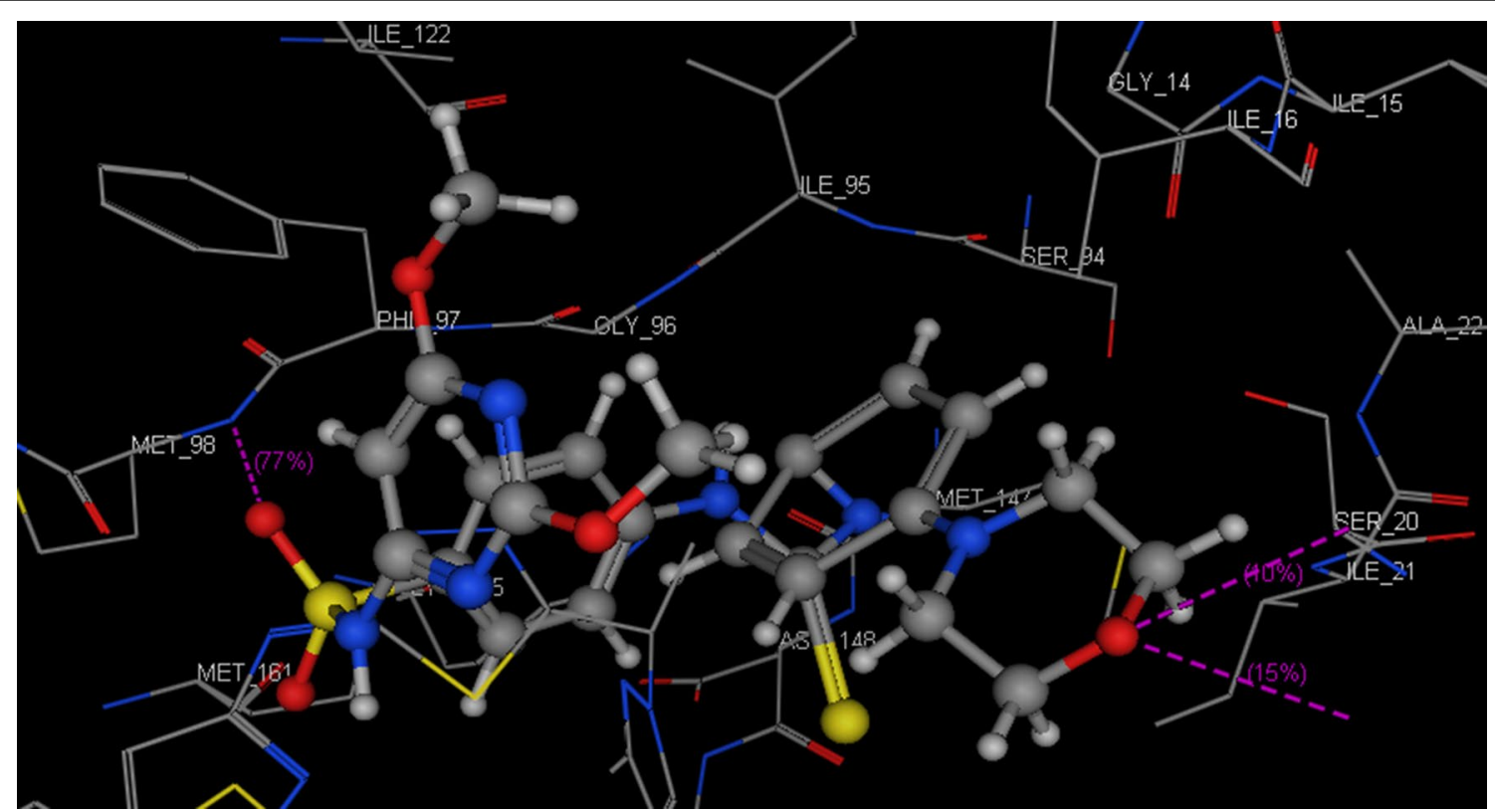

Fig. $\mathbf{5} 3 \mathrm{D}$ docking of compound $\mathbf{3 s}(S=-11.64 \mathrm{kcal} / \mathrm{mol})$ in the active site of $\mathbf{5} \mathbf{J F O}$ 
Table 3 Docking results of the targeted compounds inside $5 \mathrm{JFO}$ active site

\begin{tabular}{|c|c|c|c|c|}
\hline Compound & $\begin{array}{l}\text { Energy score (S) } \\
\text { (Kcal/mol) }\end{array}$ & $\begin{array}{l}\text { Amino } \\
\text { acids }\end{array}$ & $\begin{array}{l}\text { Interacting } \\
\text { groups }\end{array}$ & $\begin{array}{l}\text { Length } \\
\text { (Å) }\end{array}$ \\
\hline \multirow[t]{2}{*}{ Ligand } & -10.44 & Met 98 & $\mathrm{C}=\mathrm{N}$ & 2.72 \\
\hline & & Met 98 & $\mathrm{NH}$ & 2.75 \\
\hline \multirow[t]{2}{*}{$3 a$} & -8.44 & Met 98 & $\mathrm{SO}_{2}$ & 3.01 \\
\hline & & Met 98 & $\mathrm{NH}$ & 2.14 \\
\hline \multirow[t]{2}{*}{$3 b$} & -8.30 & Met 98 & $\mathrm{SO}_{2}$ & 2.17 \\
\hline & & Met 98 & $\mathrm{NH}$ & 3.12 \\
\hline \multirow[t]{2}{*}{$3 c$} & -7.89 & Met 98 & $\mathrm{SO}_{2}$ & 2.63 \\
\hline & & Met 98 & $\mathrm{NH}$ & 2.35 \\
\hline \multirow[t]{2}{*}{ 3d } & -9.02 & Met 98 & $\mathrm{SO}_{2}$ & 1.78 \\
\hline & & Met 98 & $\mathrm{NH}$ & 2.54 \\
\hline \multirow[t]{3}{*}{$3 e$} & -8.80 & Met 98 & $\mathrm{SO}_{2}$ & 1.90 \\
\hline & & Met 98 & $\mathrm{NH}$ & 2.31 \\
\hline & & Ser 19 & $\mathrm{CO}$ & 2.76 \\
\hline \multirow[t]{2}{*}{$3 f$} & -8.32 & Met 98 & $\mathrm{SO}_{2}$ & 3.13 \\
\hline & & Met 98 & $\mathrm{NH}$ & 2.85 \\
\hline \multirow[t]{2}{*}{$3 g$} & -7.92 & Met 98 & $\mathrm{SO}_{2}$ & 2.12 \\
\hline & & Met 98 & $\mathrm{NH}$ & 2.90 \\
\hline \multirow[t]{2}{*}{$3 \mathbf{i}$} & -9.07 & Met 98 & $\mathrm{SO}_{2}$ & 1.72 \\
\hline & & Met 98 & $\mathrm{NH}$ & 2.44 \\
\hline \multirow[t]{2}{*}{31} & -7.91 & Met 98 & $\mathrm{SO}_{2}$ & 2.41 \\
\hline & & Met 98 & $\mathrm{NH}$ & 3.04 \\
\hline \multirow[t]{3}{*}{$3 n$} & -9.00 & Met 98 & $\mathrm{SO}_{2}$ & 2.31 \\
\hline & & Met 98 & $\mathrm{NH}$ & 2.82 \\
\hline & & Thr 17 & $\mathrm{~N}-\mathrm{CH}_{3}$ & 3.08 \\
\hline \multirow[t]{3}{*}{30} & -8.42 & Met 98 & $\mathrm{SO}_{2}$ & 2.89 \\
\hline & & Met 98 & $\mathrm{NH}$ & 3.10 \\
\hline & & Ser 20 & $\mathrm{CO}$ & 1.99 \\
\hline \multirow[t]{3}{*}{$3 p$} & -8.98 & Met 98 & $\mathrm{SO}_{2}$ & 2.67 \\
\hline & & Met 98 & $\mathrm{NH}$ & 2.71 \\
\hline & & Ser 19 & $\mathrm{CO}$ & 3.05 \\
\hline \multirow[t]{4}{*}{$3 r$} & -8.14 & Met 98 & $\mathrm{SO}_{2}$ & 1.83 \\
\hline & & Met 98 & $\mathrm{NH}$ & 2.98 \\
\hline & & Ser 19 & N (triazole) & 3.02 \\
\hline & & Thr 17 & N (triazole) & 2.56 \\
\hline \multirow[t]{3}{*}{$3 s$} & -11.64 & Met 98 & $\mathrm{SO}_{2}$ & 2.15 \\
\hline & & Met 98 & $\mathrm{NH}$ & 2.65 \\
\hline & & Ser 20 & $\mathrm{CO}$ & 3.10 \\
\hline \multirow[t]{2}{*}{ 3t } & -7.88 & Met 98 & $\mathrm{SO}_{2}$ & 2.73 \\
\hline & & Met 98 & $\mathrm{NH}$ & 2.84 \\
\hline
\end{tabular}

\section{SAR (structure activity relationship)}

From the results revealed by the antimycobacterial activity and the docking study, it is apparent that the group attached to the thiourea is crucial for the activity. The benzo[1,3]dioxol derivative $3 \mathbf{i}(\mathrm{MIC}=6.4 \mu \mathrm{M})$ was the most potent, followed by the 4-morpholinyl-4-phenyl derivative $3 \mathrm{~s}(\mathrm{MIC}=11.8 \mu \mathrm{M})$, the oxygen atom of morpholine binds to Ser 20 inside the active site. Also, $3 \mathbf{i}$ and 3s have shown similar binding to that of the cocrystallized ligand inside the active site of $M$. tuberculosis enoyl reductase InhA and the best binding score in this series. The dipyridinyl-[1,2,4] triazole $3 \mathbf{r}$ and the 2-methyl1,3-dioxo-2,3-dihydro- $1 \mathrm{H}$-isoindole derivative $\mathbf{3 q}$ also showed potent activity, with MIC $=21.7$ and $43.5 \mu \mathrm{M}$, respectively. It is apparent that the nitrogens of the triazole ring in $3 \mathbf{r}$ tend to make additional binding interactions inside the active site of the enzyme as well as the carbonyl group in 3q, which may contribute to their antimycobacterial activity.

\section{Experimental \\ Chemistry}

All analyses were performed at King Saud University Research Center (Riyadh, Saudi Arabia). Melting points were determined in open capillaries on a Gallenkamp melting point apparatus (Sanyo Gallenkamp, Southborough, UK). Precoated silica gel plates (Kieselgel $0.25 \mathrm{~mm}$, 60 F254, Merck, Darmstadt, Germany) were used for thin layer chromatography using a developing solvent system of 4:1 chloroform/methanol and the spots were detected by the ultraviolet lamp. IR spectra ( $\mathrm{KBr}$ discs) were recorded using an FT-IR spectrometer (Perkin Elmer, Waltham, MA, USA). ${ }^{1} \mathrm{H}$-NMR spectra were scanned on NMR spectrometer (Bruker AXS Inc., Flawil, Switzerland), operating at $500 \mathrm{MHz}$ for ${ }^{1} \mathrm{H}$ and $125.76 \mathrm{MHz}$ for ${ }^{13} \mathrm{C}$. Chemical shifts are expressed in $\delta$ values (ppm) relative to TMS as an internal standard, using DMSO-d6 as a solvent. Mass spectra were recorded on a $600 \mathrm{GC} / \mathrm{MS}$ (Clarus, Middletown, CT, USA) and TQ 320 GC/MS/MS mass spectrometers (Varian, West Sussex, UK). Elemental analyses were done on a model $2400 \mathrm{CHNSO}$ analyzer (Perkin Elmer, Waltham, MA, USA). All reagents used were of the analytical grade.

\section{General method for the synthesis $\mathrm{N}$-(2,6-dimethoxypyrimidin-4-yl)-4-(3-(aryl)thioureido) benzenesulfonamides $3 a-t$}

A mixture of isothiocyanatobenzenesulfonamide 2 [27] $(0.01 \mathrm{~mol})$ with a heterocyclic amine $(0.01 \mathrm{~mol})$ was refluxed in dioxane $(30 \mathrm{~mL})$ containing triethylamine $(0.1 \mathrm{~mL})$ for $1 \mathrm{~h}$. The solvent was evaporated, the solid obtained was washed with petroleum ether (bp $40-60^{\circ} \mathrm{C}$ ) and crystallized from ethanol to afford the thiourea derivatives.

N-(2,6-Dimethoxy-pyrimidin-4-yl)-4-(3-(2-methyl-4-nitro-phenyl)thioureido)benzenesulfonamide (3a) This compound was obtained as yellow powder from ethanol; yield 84\%; m.p. $137.9^{\circ} \mathrm{C}$. IR: 3471, 3363, $3230(\mathrm{NH}), 3100$ (arom.), 2983, 2817 (aliph.), $1633(\mathrm{CN}), 1396,1151\left(\mathrm{SO}_{2}\right)$, 
1290 (CS). ${ }^{1} \mathrm{H}-\mathrm{NMR}: \delta 2.1$ [s, 3H, $\left.\mathrm{CH}_{3}\right], 3.79,3.84[2 \mathrm{~s}, 6 \mathrm{H}$, $2 \mathrm{OCH}_{3}$ ], 6.5 [s, $1 \mathrm{H}, \mathrm{H}$-pyrimidine], 6.8-7.9 [m, 7H, Ar-H], $9.8\left[\mathrm{~s}, 1 \mathrm{H}, \mathrm{SO}_{2} \mathrm{NH}\right], 11.4[\mathrm{~s}, 2 \mathrm{H}, 2 \mathrm{NH}] ;{ }^{13} \mathrm{C}-\mathrm{NMR}: 17.4,55.4$ (2), 80.6, 112.7, 120.7, 124.5 (2), 126.6, 128.4 (2), 133.0, 139.8, 141.3, 143.8 (2), 154.4, 162.6, 169.0, 178.5. Anal. Calcd. for $\mathrm{C}_{20} \mathrm{H}_{22} \mathrm{~N}_{6} \mathrm{O}_{6} \mathrm{~S}_{2}$ : C, $47.42 \% ; \mathrm{H}, 4.38 \%$; N, $16.59 \% ; \mathrm{S}, 12.66 \%$. Found: C, 47.40\%; H, 4.30\%; N, 16.50\%; S, 12.60\%.

N-(2,6-Dimethoxy-pyrimidin-4-yl)-4-(3-(2-methyl-6-nitro-phenyl)thioureido) benzenesulfonamide (3b) This compound was obtained as yellow powder from ethanol; yield 82\%; m.p. $199.3{ }^{\circ} \mathrm{C}$. IR: 3458, $3371(\mathrm{NH}), 3100$ (arom.), 2970, 2831 (aliph.), 1622 (CN), 1392, $1130\left(\mathrm{SO}_{2}\right)$, 1251 (CS). ${ }^{1} \mathrm{H}-\mathrm{NMR}: \delta 2.1\left[\mathrm{~s}, 3 \mathrm{H}, \mathrm{CH}_{3}\right], 3.81,3.84[2 \mathrm{~s}$, $\left.6 \mathrm{H}, 2 \mathrm{OCH}_{3}\right], 6.1[\mathrm{~s}, 1 \mathrm{H}, \mathrm{H}$-pyrimidine], $6.8-8.0[\mathrm{~m}, 7 \mathrm{H}$, $\mathrm{Ar}-\mathrm{H}], 8.8\left[\mathrm{~s}, 1 \mathrm{H}, \mathrm{SO}_{2} \mathrm{NH}\right], 11.5,11.9[2 \mathrm{~s}, 2 \mathrm{H}, 2 \mathrm{NH}]$. ${ }^{13}$ C-NMR: $18.3,53.4,53.6,83.6,121.7,123.8$ (2), 126.2 (2), 131.2 (2), 136.5(2), 136.9, 141.2, 144.8, 155.6, 167.1, 172.8, 186.3. Anal. Calcd. for $\mathrm{C}_{20} \mathrm{H}_{22} \mathrm{~N}_{6} \mathrm{O}_{6} \mathrm{~S}_{2}: \mathrm{C}, 47.42 \% ; \mathrm{H}$, 4.38\%; N, 16.59\%; S, $12.66 \%$. Found: C, 47.40\%; H, 4.30\%; $\mathrm{N}, 16.50 \% ; \mathrm{S}, 12.60 \%$.

4-(3-(3,5-Dimethoxyphenyl)thioureido)-N-(2,6-dimethoxy-pyrimidin-4-yl)benzenesulfonamide (3c) This compound was obtained as yellow powder from ethanol; yield 80\%; m.p. $179.6{ }^{\circ} \mathrm{C}$. IR: 3437, 3210 (NH), 3100 (arom.), 2920, 2848 (aliph.), 1622 (CN), 1354, $1153\left(\mathrm{SO}_{2}\right), 1274$ (CS). ${ }^{1} \mathrm{H}-\mathrm{NMR}: \delta 3.81,3.82\left[2 \mathrm{~s}, 6 \mathrm{H}, 2 \mathrm{OCH}_{3}\right.$, pyrimidine], 3.84, $3.88\left[2 \mathrm{~s}, 6 \mathrm{H}, 2 \mathrm{OCH}_{3}\right], 5.6[2 \mathrm{~s}, 3 \mathrm{H}, \mathrm{CH}$, dimethoxyphenyl], 6.2 [s, $1 \mathrm{H}, \mathrm{H}$-pyrimidine], 6.9-8.1 [m, 4H, Ar-H], 8.9 [s, $1 \mathrm{H}, \mathrm{SO}_{2} \mathrm{NH}$ ], 9.7 [s, $2 \mathrm{H}, 2 \mathrm{NH}$ ]; ${ }^{13} \mathrm{C}-\mathrm{NMR}: 55.1,55.4,55.5$ (2), 79.4, 98.7, 112.7 (2), 128.1 (2), 131.7 (2), 134.6, 140.7, 142.3, 158.7 (2), 159.1, 163.8, 174.6, 192.4. Anal. Calcd. For $\mathrm{C}_{21} \mathrm{H}_{23} \mathrm{~N}_{5} \mathrm{O}_{6} \mathrm{~S}_{2}: \mathrm{C}, 49.89 \%$; $\mathrm{H}, 4.59 \%$; N, $13.85 \%$; $\mathrm{S}, 12.68 \%$. Found: C, $49.80 \%$; H, 4.50\%; N, 13.80\%; S, $12.60 \%$.

$N-(2,6-D i m e t h o x y-p y r i m i d i n-4-y l)-4-(3-(3,4,5-$ trimethoxyphenyl)thioureido) benzenesulfonamide (3d) This compound was obtained as Brown powder from ethanol; yield 87\%; m.p. $275.5^{\circ} \mathrm{C}$. IR: 3433, $3356(\mathrm{NH}), 3053$ (arom.), 2939, 2835 (aliph.), 1616 (CN), 1354, $1128\left(\mathrm{SO}_{2}\right)$, 1276 (CS). ${ }^{1} \mathrm{H}-\mathrm{NMR}: \delta 3.61,3.67\left[2 \mathrm{~s}, 6 \mathrm{H}, 2 \mathrm{O} \mathrm{CH}_{3}\right]$, $3.81,3.84\left[2 \mathrm{~s}, 9 \mathrm{H}, 3 \mathrm{OCH}_{3}\right], 5.9[\mathrm{~s}, 2 \mathrm{H}, \mathrm{Ar}-\mathrm{H}], 6.4[\mathrm{~s}$, $1 \mathrm{H}, \mathrm{H}$-pyrimidine], 6.9-8.3 [m, $4 \mathrm{H}, \mathrm{Ar}-\mathrm{H}], 9.7[\mathrm{~s}, 1 \mathrm{H}$, $\left.\mathrm{SO}_{2} \mathrm{NH}\right], 11.8[\mathrm{~s}, 2 \mathrm{H}, 2 \mathrm{NH}] .{ }^{13} \mathrm{C}-\mathrm{NMR}: 55.2,55.6,55.9(2)$, 63.7, 83.5, 98.0 (2), 121.6 (2), 128.3 (2), 132.1, 133.7, 135.2, 140.6, 156.0 (2), 158.8, 166.4, 170.3, 181.1. Anal. Calcd. for $\mathrm{C}_{22} \mathrm{H}_{25} \mathrm{~N}_{5} \mathrm{O}_{7} \mathrm{~S}_{2}: \mathrm{C}, 49.34 \% ; \mathrm{H}, 4.70 \%$; N, $13.08 \% ; \mathrm{S}, 11.97 \%$. Found: C, 49.34\%; H, 4.70\%; N, 13.08\%; S, 11.97\%.

N-(2,6-Dimethoxy-pyrimidin-4-yl)-4-(3-(4-ethoxyphenyl) thioureido) benzenesulfonamide (3e) This compound was obtained as Grey powder from ethanol; yield 83\%; m.p. $234.2^{\circ} \mathrm{C}$. IR: 3296,3217 (NH), 3100 (arom.), 2978, 2929, 2873 (aliph.), 1639 (CN), 1390, $1168\left(\mathrm{SO}_{2}\right), 1246$ (CS). ${ }^{1} \mathrm{H}-\mathrm{NMR}: \delta 1.2\left[\mathrm{t}, 3 \mathrm{H}, \mathrm{CH}_{3}, J=8 \mathrm{~Hz}\right.$ ] $3.90,3.92$ $\left[2 \mathrm{~s}, 6 \mathrm{H}, 2 \mathrm{OCH}_{3}\right], 4.0\left[\mathrm{q}, 2 \mathrm{H}, \mathrm{CH}_{2}\right], 6.8[\mathrm{~s}, 1 \mathrm{H}, \mathrm{H}$-pyrimidine $], 7.0-8.4[\mathrm{~m}, 8 \mathrm{H}, \mathrm{Ar}-\mathrm{H}], 9.5\left[\mathrm{~s}, 1 \mathrm{H}, \mathrm{SO}_{2} \mathrm{NH}\right]$, 11.7 [s, 2H, 2NH]. ${ }^{13} \mathrm{C}-\mathrm{NMR}: 15.2,53.2,53.9,63.5$, 84.6, 115.0 (2), 120.3 (2), 127.9 (2), 128.3 (2), 133.3 (2), 142.1, 153.4, 154.0, 164.8, 172.6, 179.3. Anal. Calcd. for $\mathrm{C}_{21} \mathrm{H}_{23} \mathrm{~N}_{5} \mathrm{O}_{5} \mathrm{~S}$ : C, $51.52 \% ; \mathrm{H}, 4.74 \% ; \mathrm{N}, 14.30 \%$; S, $13.10 \%$. Found: C, 51.50\%; H, 4.70\%; N, 14.30\%; S, $13.10 \%$.

4-(3-Benzyl-thioureido)-N-(2,6-dimethoxy-pyrimidin-4-yl)benzenesulfonamide (3f) This compound was obtained as yellow powder from ethanol; yield 88\%; m.p. > $360{ }^{\circ} \mathrm{C}$. IR: 3365, 3188 (NH), 3034 (arom.), 2981, 2827 (aliph.), $1622(\mathrm{CN}), 1390,1128\left(\mathrm{SO}_{2}\right), 1251$ (CS). ${ }^{1} \mathrm{H}-\mathrm{NMR}: \delta 3.61,3.64\left[2 \mathrm{~s}, 6 \mathrm{H}, 2 \mathrm{OCH}_{3}\right], 4.3\left[\mathrm{~s}, 2 \mathrm{H}, \mathrm{CH}_{2}\right]$, $6.4[\mathrm{~s}, 1 \mathrm{H}, \mathrm{CH}$ pyrimidine], 7.0-8.5 [m, 9H, $\mathrm{Ar}-\mathrm{H}], 9.9$ [s, $\left.1 \mathrm{H}, \mathrm{SO}_{2} \mathrm{NH}\right], 10.8,11.7$ [2s, $\left.2 \mathrm{H}, 2 \mathrm{NH}\right] .{ }^{13} \mathrm{C}-\mathrm{NMR}$ : 49.2, 53.4, 53.8, 85.6, 120.8 (2), 125.7, 127.3 (2), 127.7 (2), 128.8 (2), 139.4 (2), 139.6, 161.5, 167.9, 171.9, 178.8. Anal. Calcd. for $\mathrm{C}_{20} \mathrm{H}_{21} \mathrm{~N}_{5} \mathrm{O}_{4} \mathrm{~S}_{2}$ : C, 52.27\%; H, 4.61\%; N, 15.24\%; S, 13.95\%. Found: C, 52.20\%; H, 4.60\%; N, 15.20\%; S, $13.90 \%$.

4-(3-(2,3-Dimethoxybenzyl)thioureido)-N-(2,6-dimethoxy-pyrimidin-4-yl) benzenesulfonamide (3g) This compound was obtained as yellow powder from ethanol; yield 88\%; m.p. $151.1{ }^{\circ} \mathrm{C}$. IR: 3292, 3181 (NH), 3047 (arom.), 2986, 2866, 2831 (aliph.), 1587 (CN), 1388, $1172\left(\mathrm{SO}_{2}\right)$, 1228 (CS). ${ }^{1} \mathrm{H}-\mathrm{NMR}: \delta 3.75,3.77,3.80$ [3s, $\left.12 \mathrm{H}, 4 \mathrm{OCH}_{3}\right]$, $4.3\left[\mathrm{~s}, 2 \mathrm{H}, \mathrm{CH}_{2}\right], 6.8$ [s, 1H, H-pyrimidine], 6.9-8.0 [m, $7 \mathrm{H}, \mathrm{Ar}-\mathrm{H}], 8.1,8.3\left[2 \mathrm{~s}, 3 \mathrm{H}, \mathrm{SO}_{2} \mathrm{NH}+2 \mathrm{NH}\right]{ }^{13} \mathrm{C}-\mathrm{NMR}$ : 40.4, 56.1 (2), 56.7, 60.5, 83.4, 112.3, 120.7 (2), 124.3 (2), 127.0, 128.1 (2), 133.1, 140.2, 146.6, 146.7, 161.4, 165.4, 169.5, 184.6. Anal. Calcd. for $\mathrm{C}_{22} \mathrm{H}_{25} \mathrm{~N}_{5} \mathrm{O}_{6} \mathrm{~S}_{2}$ : C, $50.86 \%$; $\mathrm{H}$, 4.85\%; N, 13.48\%; S, 12.34\%. Found: C, 50.80\%; H, 4.80\%; N, 13.40\%; S, 12.30\%.

N-(2,6-Dimethoxy-pyrimidin-4-yl)-4-(3-phenethyl-thioureido)benzenesulfonamide (3h) This compound was obtained as yellow powder from ethanol; yield 85\%; m.p. $189.7^{\circ} \mathrm{C}$. IR: 3367, 3238 (NH), 3100 (arom.), 2981, 2811 (aliph.), 1635 (CN), 1394, $1130\left(\mathrm{SO}_{2}\right), 1274$ (CS). ${ }^{1} \mathrm{H}-\mathrm{NMR}: \delta 2.7,3.5$ [2t, $\left.4 \mathrm{H}, 2 \mathrm{CH}_{2}, J=8 \mathrm{~Hz}\right], 3.63,3.65$ [2s, $\left.6 \mathrm{H}, 2 \mathrm{OCH}_{3}\right], 6.6[\mathrm{~s}, 1 \mathrm{H}, \mathrm{H}$-pyrimidine], 7.1-8.1 [m, $9 \mathrm{H}, \mathrm{Ar}-\mathrm{H}], 9.7\left[\mathrm{~s}, 1 \mathrm{H}, \mathrm{SO}_{2} \mathrm{NH}\right], 11.0[\mathrm{~s}, 2 \mathrm{H}, 2 \mathrm{NH}] .{ }^{13} \mathrm{C}-$ NMR: 35.4, 40.4, 55.6, 55.9, 85.0, 124.1 (2), 126.6, 128.8 (2), 129.0 (2), 129.7 (2), 132.7, 140.1, 142.3, 161.5, 165.4, 170.2, 181.8. Anal. Calcd. for $\mathrm{C}_{21} \mathrm{H}_{23} \mathrm{~N}_{5} \mathrm{O}_{4} \mathrm{~S}_{2}$ : C, 53.26\%; H, 4.90\%; N, 14.79\%; S, 13.54\%. Found: C, 53.26\%; H, 4.90\%; N, 14.79\%; S, 13.54\%. 
4-(3-Benzo[1,3]dioxol-5-yl-thioureido)-N-(2,6-dimethoxy-pyrimidin-4-yl)benzenesulfonamide (3i) This compound was obtained as yellow powder from ethanol; yield 85\%; m.p. $247.1{ }^{\circ} \mathrm{C}$. IR: 3385, 3169 (NH), 3064 (arom.), 2910, 2895 (aliph.), 1618 (CN), 1381, $1124\left(\mathrm{SO}_{2}\right), 1240$ (CS). ${ }^{1} \mathrm{H}-\mathrm{NMR}: \delta 3.82,3.84\left[2 \mathrm{~s}, 6 \mathrm{H}, 2 \mathrm{OCH}_{3}\right], 5.9[\mathrm{~s}, 1 \mathrm{H}$, $\mathrm{H}$-pyrimidine], 6.0 [s, 2H, O- $\left.\mathrm{CH}_{2}-\mathrm{O}\right], 6.7-8.4[\mathrm{~m}, 7 \mathrm{H}$, $\mathrm{Ar}-\mathrm{H}], 9.8\left[\mathrm{~s}, 1 \mathrm{H}, \mathrm{SO}_{2} \mathrm{NH}\right], 10.7,11.4[2 \mathrm{~s}, 2 \mathrm{H}, 2 \mathrm{NH}] .{ }^{13} \mathrm{C}-$ NMR: 55.4, 56.5, 83.7, 101.2, 109.3, 113.6, 118.2, 120.9 (2), 128.8 (2), 129.1, 136.7, 142.6, 146.8, 148.2, 152.9, 167.5, 172.4, 182.0. Anal. Calcd. for $\mathrm{C}_{20} \mathrm{H}_{19} \mathrm{~N}_{5} \mathrm{O}_{6} \mathrm{~S}_{2}: \mathrm{C}, 49.07 \% ; \mathrm{H}$, 3.91\%; N, $14.31 \%$; S, $13.10 \%$. Found: C, $49.00 \%$;, $3.90 \%$; $\mathrm{N}, 14.30 \%$;, $13.10 \%$.

4-(3-Benzo[1,3]dioxol-4-yl-methyl-thioureido)-N-(2,6-di methoxy-pyrimidin-4-yl)benzenesulfonamide (3j) This compound was obtained as yellow powder from ethanol; yield 89\%; m.p. > $360^{\circ} \mathrm{C}$. IR: 3410, $3371(\mathrm{NH}), 3100$ (arom.), 2966, 2889 (aliph.), $1553(\mathrm{CN}), 1376,1128\left(\mathrm{SO}_{2}\right)$, 1251 (CS). ${ }^{1} \mathrm{H}-\mathrm{NMR}: \delta 3.61,3.64\left[2 \mathrm{~s}, 6 \mathrm{H}, 2 \mathrm{OCH}_{3}\right], 4.2[\mathrm{~s}$, $2 \mathrm{H}, \mathrm{CH}_{2} \mathrm{NH}$ ], 6.0 [s, 2H, O- $\mathrm{CH}_{2}-\mathrm{O}$ ], 6.6 [s, 1H, H-pyrimidine], 7.0-8.5 [m, 7H, Ar-H], 9.9 [s, $\left.1 \mathrm{H}, \mathrm{SO}_{2} \mathrm{NH}\right], 10.3$, 12.6 [2s, $2 \mathrm{H}, 2 \mathrm{NH}] ;{ }^{13} \mathrm{C}-\mathrm{NMR}: 52.4,54.3,54.6,82.4,101.3$, 108.5, 108.9, 120.6, 124.6 (2), 128.9 (2), 129.7, 133.2, 141.0, 143.9, 146.7, 160.5, 161.7, 163.6, 173.0. Anal. Calcd. for $\mathrm{C}_{21} \mathrm{H}_{21} \mathrm{~N}_{5} \mathrm{O}_{6} \mathrm{~S}_{2}$ : C, 50.09\%; H, 4.20\%; N, $13.91 \%$; S, $12.73 \%$. Found: C, $50.09 \%$; H, 4.20\%; N, 13.90\%; S, 12.70\%.

N-(2,6-Dimethoxy-pyrimidin-4-yl)-4-(3-naphthalen-1-yl-thioureido)benzenesulfonamide (3k) This compound was obtained as yellow powder from ethanol; yield 81\%; m.p. $152.0^{\circ} \mathrm{C}$. IR: 3354, $3232(\mathrm{NH}), 3051$ (arom.), 2951, 2836 (aliph.), $1620(\mathrm{CN}), 1394,1184\left(\mathrm{SO}_{2}\right)$, 1247 (CS). ${ }^{1} \mathrm{H}-\mathrm{NMR}: \delta 3.83,3.86\left[2 \mathrm{~s}, 6 \mathrm{H}, 2 \mathrm{OCH}_{3}\right.$ ], 5.9 [s, $1 \mathrm{H}, \mathrm{H}$-pyrimidine], 6.9-8.4 [m, $11 \mathrm{H}, \mathrm{Ar}-\mathrm{H}], 9.8[\mathrm{~s}, 1 \mathrm{H}$, $\left.\mathrm{SO}_{2} \mathrm{NH}\right], 11.8[\mathrm{~s}, 2 \mathrm{H}, 2 \mathrm{NH}] .{ }^{13} \mathrm{C}-\mathrm{NMR}: 55.3,56.5,86.0$, 107.9, 115.8, 122.7, 123.2 (2), 124.1, 125.9, 126.3, 127.1, 127.9, 128.2 (2), 130.0, 134.6, 143.1 (2), 163.7, 165.5, 173.6, 181.0. Anal. Calcd. for $\mathrm{C}_{23} \mathrm{H}_{21} \mathrm{~N}_{5} \mathrm{O}_{4} \mathrm{~S}_{2}$ : C, 55.74\%; $\mathrm{H}$, 4.27\%; N, 14.13\%; S, $12.94 \%$. Found: C, $55.70 \%$; H, $4.20 \%$; $\mathrm{N}, 14.10 \%$;, $12.90 \%$.

N-(2,6-Dimethoxy-pyrimidin-4-yl)-4-(3-(5,6,7,8-tetrahydro-naphthalen-1-yl)thioureido)benzenesulfonamide (3l) This compound was obtained as brown powder from ethanol; yield 83\%; m.p. $257.9{ }^{\circ} \mathrm{C}$. IR: 3400,3309 (NH), 3082 (arom.), 2931, 2835 (aliph.), 1616 (CN), 1377, $1136\left(\mathrm{SO}_{2}\right), 1280(\mathrm{CS}) .{ }^{1} \mathrm{H}-\mathrm{NMR}: \delta 1.6-2.8\left[\mathrm{~m}, 8 \mathrm{H}, 4 \mathrm{CH}_{2}\right.$ cyclo], 3.81, 3.84 [2s, $\left.6 \mathrm{H}, 2 \mathrm{OCH}_{3}\right], 6.2[\mathrm{~s}, 1 \mathrm{H}, \mathrm{H}$-pyrimidine $], 6.6-8.0[\mathrm{~m}, 7 \mathrm{H}, \mathrm{Ar}-\mathrm{H}], 8.8\left[\mathrm{~s}, 1 \mathrm{H}, \mathrm{SO}_{2} \mathrm{NH}\right], 11.7,11.9$ [2s, $2 \mathrm{H}, 2 \mathrm{NH}] .{ }^{13} \mathrm{C}-\mathrm{NMR}: 22.6$ (2), 23.3, 29.8, 55.1, 55.6, 82.7, 117.4, 119.5 (2), 120.8, 124.0, 127.4 (2), 137.1, 137.5, 137.6 (2), 146.5, 161.1, 161.8, 170.9, 179.8. Anal. Calcd. for
$\mathrm{C}_{23} \mathrm{H}_{25} \mathrm{~N}_{5} \mathrm{O}_{4} \mathrm{~S}_{2}$ : C, 55.29\%; H, 5.04\%; N, 14.02\%; S, $12.84 \%$. Found: C, $55.20 \%$; H, 5.00\%; N, 14.00\%; S, 12.80\%.

N-(2,6-Dimethoxy-pyrimidin-4-yl)-4-(3-indan-5-yl-thioureido)benzenesulfonamide (3m) This compound was obtained as yellow powder from ethanol; yield 80\%; m.p. $119.1^{\circ} \mathrm{C}$. IR: 3458, 3253 (NH), 3061 (arom.), 2945, 2889, 2839 (aliph.), 1627 (CN), 1396, $1130\left(\mathrm{SO}_{2}\right), 1273(\mathrm{CS}) .{ }^{1} \mathrm{H}-$ NMR: $\delta 1.9-2.0\left[\mathrm{~m}, 2 \mathrm{H}, \mathrm{CH}_{2}\right.$ cyclo], $2.7-2.8\left[\mathrm{~m}, 4 \mathrm{H}, 2 \mathrm{CH}_{2}\right.$ cyclo], 3.79, 3.80 [2s, $\left.6 \mathrm{H}, 2 \mathrm{OCH}_{3}\right], 5.8$ [s, $1 \mathrm{H}, \mathrm{H}$-pyrimidine], 6.6-8.4 [m, 7H, Ar-H], $9.9\left[\mathrm{~s}, 1 \mathrm{H}, \mathrm{SO}_{2} \mathrm{NH}\right], 11.4$, 12.3 [2s, 2H, 2NH]. ${ }^{13} \mathrm{C}-\mathrm{NMR}: 25.6,32.2(2), 55.4,56.5$, 79.8, 116.8 (2), 124.6 (2), 124.9, 128.3 (2), 137.2, 138.4, 144.6 (2), 145.7, 154.8, 170.0, 172.4, 183.0. Anal. Calcd. for $\mathrm{C}_{22} \mathrm{H}_{23} \mathrm{~N}_{5} \mathrm{O}_{4} \mathrm{~S}_{2}$ : C, 54.42\%; H, 4.77\%; N, 14.42\%; S, $13.21 \%$. Found: C, 54.40\%; H, 4.70\%; N, 14.40\%; S, $13.20 \%$.

N-(2,6-Dimethoxy-pyrimidin-4-yl)-4-(3-(2-(1-methyl -1H-pyrrol-2-yl)ethyl)thioureido)benzenesulfonamide (3n) This compound was obtained as brown powder from ethanol; yield 86\%; m.p. > $360{ }^{\circ} \mathrm{C}$. IR: 3410,3216 (NH), 3100 (arom.), 2943, 2839 (aliph.), 1595 (CN), 1386, $1126\left(\mathrm{SO}_{2}\right), 1262(\mathrm{CS}) .{ }^{1} \mathrm{H}-\mathrm{NMR}: \delta 2.8\left[\mathrm{t}, 2 \mathrm{H}, \mathrm{CH}_{2}\right.$, $J=8 \mathrm{~Hz}], 3.5\left[\mathrm{~s}, 3 \mathrm{H}, \mathrm{N}-\mathrm{CH}_{3}\right], 3.70,3.72\left[2 \mathrm{~s}, 6 \mathrm{H}, 2 \mathrm{OCH}_{3}\right]$, $3.9\left[\mathrm{t}, 2 \mathrm{H}, \mathrm{CH}_{2}-\mathrm{NH}, J=8 \mathrm{~Hz}\right], 5.9-6.7[\mathrm{~m}, 3 \mathrm{H}, 3 \mathrm{H}-\mathrm{pyr}-$ role], 6.8 [s, $1 \mathrm{H}, \mathrm{H}$-pyrimidine], 7.0-8.2 [m, 4H, Ar-H], $9.9\left[\mathrm{~s}, 1 \mathrm{H}, \mathrm{SO}_{2} \mathrm{NH}\right], 11.2,12.3[2 \mathrm{~s}, 2 \mathrm{H}, 2 \mathrm{NH}] .{ }^{13} \mathrm{C}-\mathrm{NMR}$ : 25.4, 35.6, 44.2, 52.9, 53.8, 83.6, 105.1, 107.3, 123.8, 123.9 (2), 125.5, 128.0 (2), 134.2, 143.1, 162.6, 163.9, 173.4, 182.3. Anal. Calcd. for $\mathrm{C}_{20} \mathrm{H}_{24} \mathrm{~N}_{6} \mathrm{O}_{4} \mathrm{~S}_{2}: \mathrm{C}, 50.41 \% ; \mathrm{H}, 5.08 \%$; N, 17.63\%; S, $13.46 \%$ Found: C, 50.40\%; H, 5.00\%; N, 17.60\%; S, $13.46 \%$.

N-(2,6-Dimethoxy-pyrimidin-4-yl)-4-(3-(2-pyrrolidin-1-yl-ethyl)thioureido) benzenesulfonamide (3o) This compound was obtained as brown powder from ethanol; yield 86\%; m.p. $280.0{ }^{\circ} \mathrm{C}$. IR: 3342, $3323(\mathrm{NH}), 3045$ (arom.), 2972, 2856 (aliph.), $1622(\mathrm{CN}), 1386,1180\left(\mathrm{SO}_{2}\right)$, 1274 (CS). ${ }^{1} \mathrm{H}-\mathrm{NMR}$ (DMSO- $d_{6}$ ): $\delta 1.6-1.8\left[\mathrm{~m}, 4 \mathrm{H}, \mathrm{CH}_{2}-\right.$ $\mathrm{CH}_{2}$-pyrrolidine], 2.51-2.56 [m, $4 \mathrm{H}, \mathrm{CH}_{2}-\mathrm{N}-\mathrm{CH}_{2}$ pyrrolidine], 2.62-2.68 [m, $2 \mathrm{H}, \mathrm{N}-\mathrm{CH}_{2}$ ], $3.4\left[\mathrm{t}, 2 \mathrm{H}, \mathrm{CH}_{2}-\mathrm{NH}\right.$, $J=8 \mathrm{~Hz}], 3.82,3.85\left[2 \mathrm{~s}, 6 \mathrm{H}, 2 \mathrm{OCH}_{3}\right], 6.5[\mathrm{~s}, 1 \mathrm{H}, \mathrm{H}$-pyrimidine], 6.9-7.9 [m, 4H, Ar-H], $9.8\left[\mathrm{~s}, 1 \mathrm{H}, \mathrm{SO}_{2} \mathrm{NH}\right], 11.4$ [s, 2H, 2NH]; ${ }^{13} \mathrm{C}-\mathrm{NMR}: 23.3$ (2), 40.4, 51.6, 52.9 (2), 53.5, 54.1, 81.7, 120.7 (2), 127.7 (2), 136.4, 140.2, 161.0, 166.3, 170.6, 180.5. Anal. Calcd. for $\mathrm{C}_{19} \mathrm{H}_{26} \mathrm{~N}_{6} \mathrm{O}_{4} \mathrm{~S}_{2}: \mathrm{C}, 48.91 \% ; \mathrm{H}$, 5.62\%; N, $18.01 \%$; S, 13.74\%. Found: C, 48.90\%; H, 5.60\%; $\mathrm{N}, 18.00 \%$;, $13.70 \%$.

N-(2,6-Dimethoxy-pyrimidin-4-yl)-4-(3-(9,10-dioxo-9,1 0-dihydro-anthracen-2-yl)thioureido)benzenesulfonamide (3p) This compound was obtained as yellow powder from ethanol; yield 84\%; m.p. $299.6{ }^{\circ} \mathrm{C}$. IR: 3433 , 3348, 
3219 (NH), 3064 (arom.), 2921, 2871 (aliph.), 1705, 1672 (2CO), 1625 (CN), 1338, 1178 ( $\left.\mathrm{SO}_{2}\right), 1280$ (CS). ${ }^{1} \mathrm{H}-\mathrm{NMR}$ : $\delta 3.70,3.73\left[2 \mathrm{~s}, 6 \mathrm{H}, 2 \mathrm{OCH}_{3}\right.$ ] 6.6 [s, $1 \mathrm{H}, \mathrm{H}$-pyrimidine], 6.9-8.4 [m, 11H, Ar-H], 10.8 [s, 1H, $\left.\mathrm{SO}_{2} \mathrm{NH}\right], 11.6,11.9$ [2s, 2H, 2NH]. ${ }^{13} \mathrm{C}-\mathrm{NMR}: 57.2,57.8,83.7,118.5,121.6$ (2), 126.7 (2), 130.0, 133.4 (2), 133.7 (2), 134.1 (2), 134.7 (2), 135.3 (2), 141.0 (2), 155.1, 162.7, 172.4, 180.5, 183.7 (2). Anal. Calcd. for $\mathrm{C}_{27} \mathrm{H}_{21} \mathrm{~N}_{5} \mathrm{O}_{6} \mathrm{~S}_{2}:$ C, 56.34\%; H, 3.68\%; N, $12.17 \%$; S, 11.14\%. Found: C, 56.30\%; H, 3.60\%; N, 12.10\%; S, $11.10 \%$.

N-(2,6-Dimethoxy-pyrimidin-4-yl)-4-(3-(2-methyl-1,3-dioxo-2,3-dihydro-1H-isoindol-5-yl)thioureido)benzenesulfonamide (3q) This compound was obtained as yellow powder from ethanol; yield $85 \%$; m.p. $194.7^{\circ} \mathrm{C}$. IR: 3475 , 3363, 3219 (NH), 3055 (arom.), 2951, 2809 (aliph.), 1755, 1689 (2CO), 1622 (CN), 1382, 1157 (SO $), 1271(\mathrm{CS}) .{ }^{1} \mathrm{H}-$ NMR: $\delta 3.0\left[\mathrm{~s}, 3 \mathrm{H}, \mathrm{N}-\mathrm{CH}_{3}\right], 3.70,3.72\left[2 \mathrm{~s}, 6 \mathrm{H}, 2 \mathrm{OCH}_{3}\right]$, $6.4[\mathrm{~s}, 1 \mathrm{H}, \mathrm{H}$-pyrimidine], 6.8- $8.0[\mathrm{~m}, 7 \mathrm{H}, \mathrm{Ar}-\mathrm{H}], 9.0[\mathrm{~s}$, $\left.1 \mathrm{H}, \mathrm{SO}_{2} \mathrm{NH}\right], 10.9$ [s, $\left.2 \mathrm{H}, 2 \mathrm{NH}\right] ;{ }^{13} \mathrm{C}-\mathrm{NMR}: 23.7,55.4,55.6$, 81.2, 117.3, 125.0 (2), 127.9 (2), 128.0 (2), 130.7, 135.0 (2), 142.1 (2), 162.7, 168.5 (2), 168.8, 174.0, 185.2. Anal. Calcd. for $\mathrm{C}_{22} \mathrm{H}_{20} \mathrm{~N}_{6} \mathrm{O}_{6} \mathrm{~S}_{2}$ : Anal. Calcd. for $\mathrm{C}_{27} \mathrm{H}_{21} \mathrm{~N}_{5} \mathrm{O}_{6} \mathrm{~S}_{2} \mathrm{C}$, 49.99\%; H, 3.81\%; N, 15.90\%; S, 12.13\%. Found: C, 49.90\%; H, 3.80\%; N, 15.90\%; S, $12.10 \%$.

N-(2,6-Dimethoxy-pyrimidin-4-yl)-4-(3-(3,5-dipyridin-2-yl- [1,2,4]triazol-4-yl)thioureido)benzenesulfonamide (3r) This compound was obtained as brown powder from ethanol; yield $83 \%$; m.p. $286.0^{\circ} \mathrm{C}$. IR: 3411 , 3188 (NH), 3100 (arom.), 2960, 2829 (aliph.), 1622 (CN), 1377, $1138\left(\mathrm{SO}_{2}\right), 1249$ (CS). ${ }^{1} \mathrm{H}-\mathrm{NMR}: \delta 3.70,3.74$ [2s, $6 \mathrm{H}, 2 \mathrm{OCH}_{3}$ ], $6.6[\mathrm{~s}, 1 \mathrm{H}, \mathrm{H}$-pyrimidine], 7.1-8.6 [m, $12 \mathrm{H}$, $\mathrm{Ar}-\mathrm{H}], 9.8$ [s, 1H, SO $\mathrm{NH}], 10.9,11.9$ [s, 2H, 2NH]. ${ }^{13} \mathrm{C}-$ NMR: 56.7, 56.9, 85.6, 121.9 (2), 125.0 (2), 126.1 (2), 128.8 (2), 137.9, 138.2 (2), 140.6, 143.1 (2), 150.0 (2), 151.6 (2), 162.8, 164.0, 174.3, 187.1. Anal. Calcd. for $\mathrm{C}_{25} \mathrm{H}_{22} \mathrm{~N}_{10} \mathrm{O}_{4} \mathrm{~S}_{2}$ : Anal. Calcd. for $\mathrm{C}_{27} \mathrm{H}_{21} \mathrm{~N}_{5} \mathrm{O}_{6} \mathrm{~S}_{2} \mathrm{C}, 50.84 \% ; \mathrm{H}, 3.75 \%$; N, 23.71\%; S, 10.86\%. Found: C, 50.80\%; H, 3.7 0\%; N, 23.70\%; S, $10.86 \%$.

N-(2,6-Dimethoxy-pyrimidin-4-yl)-4-(3-(4-morpholin-4-yl-phenyl)thioureido)benzenesulfonamide(3s) This compound was obtained as brown powder from ethanol; yield 81\%; m.p. $184.0^{\circ} \mathrm{C}$. IR: 3377, $3304(\mathrm{NH}), 3069$ (arom.), 2962, 2852 (aliph.), 1635 (CN), 1379, 1151 $\left(\mathrm{SO}_{2}\right), 1263$ (CS). ${ }^{1} \mathrm{H}-\mathrm{NMR}$ : $\delta$ 3.1-3.2 [m, 4H, $\mathrm{CH}_{2}-\mathrm{N}-$ $\mathrm{CH}_{2}$ ] $3.60-3.68\left[\mathrm{~m}, 4 \mathrm{H}, \mathrm{CH}_{2}-\mathrm{O}-\mathrm{CH}_{2}\right], 3.79,3.85$ [2s, $6 \mathrm{H}, 2 \mathrm{OCH}_{3}$ ], $5.9[\mathrm{~s}, 1 \mathrm{H}, \mathrm{H}$-pyrimidine], 6.5-8.3 [m, $8 \mathrm{H}$, $\mathrm{Ar}-\mathrm{H}], 9.9\left[\mathrm{~s}, 1 \mathrm{H}, \mathrm{SO}_{2} \mathrm{NH}\right], 10.6,11.0[2 \mathrm{~s}, 2 \mathrm{H}, 2 \mathrm{NH}] .{ }^{13} \mathrm{C}-$ NMR: 49.7 (2), 55.4, 55.6, 66.6 (2), 84.0, 112.9 (2), 119.8 (2), 124.9 (2), 127.6, 129.8 (2), 133.7, 140.0, 146.8, 155.9, 167.0, 173.4, 179.7. Anal. Calcd. for $\mathrm{C}_{23} \mathrm{H}_{26} \mathrm{~N}_{6} \mathrm{O}_{5} \mathrm{~S}_{2}: \mathrm{C}$,
52.06\%; H, 4.94\%; N, 15.84\%; S, 12.09\%. Found: C, 52.00\%; H, $4.90 \%$; N, $15.80 \%$; S, $12.00 \%$.

4-(3-Adamantan-1-yl-thioureido)-N-(2,6-dimethoxy-pyrimidin-4-yl) benzenesulfonamide (3t) This compound was obtained as white crystals from ethanol; yield 81\%; m.p. $164.6{ }^{\circ} \mathrm{C}$. IR: 3346, 3176 (NH), 3100 (arom.), 2910, 2852 (aliph.), 1625 (CN), 1375, $1186\left(\mathrm{SO}_{2}\right), 1236$ (CS). ${ }^{1} \mathrm{H}-\mathrm{NMR}: \delta 1.6-1.7$ [m, 3H, 3CH-adamantyl], 1.8-2.1 [m, $12 \mathrm{H}, 6 \mathrm{CH}_{2}$-adamantyl], 3.80, $3.83\left[2 \mathrm{~s}, 6 \mathrm{H}, 2 \mathrm{OCH}_{3}\right]$, 5.9 [s, 1H, H-pyrimidine], 7.1-8.4 [m, 4H, Ar-H], 9.8 [s, $\left.1 \mathrm{H}, \mathrm{SO}_{2} \mathrm{NH}\right], 11.4,12.6[2 \mathrm{~s}, 2 \mathrm{H}, 2 \mathrm{NH}] .{ }^{13} \mathrm{C}-\mathrm{NMR}: 29.4$ (3), 35.2 (3), 42.5 (3), 43.4, 59.2, 63.4, 85.3, 120.6 (2), 130.7 (2), 136.8, 142.1, 153.9, 169.5, 172.8, 182.1. Anal. Calcd. for $\mathrm{C}_{23} \mathrm{H}_{29} \mathrm{~N}_{5} \mathrm{O}_{4} \mathrm{~S}_{2}$ : C, 54.85\%; H, 5.80\%; N, 13.91\%; S, $12.73 \%$. Found: C, 54.80\%; H, 5.80\%; N, 13.90\%; S, $12.70 \%$.

\section{Antimycobacterial activity}

The M. tuberculosis (RCMB 010126) strain was provided from the culture collection of the Regional Center for Mycology and Biotechnology (RCMB), Al-Azhar University (Cairo, Egypt). The antimycobacterial activity of the synthesized compounds was performed using the microplate Alamar blue assay technique [35] with minor modifications which were performed in sterile 96 well microplates. To prevent dehydration in experimental wells, the outer perimeter wells of the plate were filled with sterile water. $100 \mu \mathrm{L}$ of $10^{5} \mathrm{CFU} / \mathrm{mL}$ M. tuberculosis inoculum was added to the wells. For detecting the antimycobacterial activity of the synthesized compounds, $100 \mu \mathrm{L}$ (at $200 \mu \mathrm{g} / \mathrm{mL}$ ) dissolved in dimethyl sulfoxide was then added to the wells. Isoniazid was used as a positive control. Also, additional control wells consisted of bacteria only was performed. Five replicates were tested for each treatment along with the controls. The plates were then incubated for at least 4 days at $37{ }^{\circ} \mathrm{C}$. After the end of incubation period, $20 \mu \mathrm{L}$ of Alamar blue solution (Alamar Biosciences/Accumed, Westlake, OH, USA) and $12.5 \mu \mathrm{L}$ of $20 \%$ Tween 80 (Merck, Darmstadt, Germany) were added to all the wells of the plate. The plates were then incubated again at $37{ }^{\circ} \mathrm{C}$ for $24 \mathrm{~h}$ in the dark. The results were recorded at $24 \mathrm{~h}$ post-reagent addition at $590 \mathrm{~nm}$. The percent of inhibition was defined as: $1-$ (mean of the test well $/$ mean of B wells $) \times 100$. For the determination of the minimum inhibitory concentrations, stock solutions of the tested compounds were prepared in dimethyl sulfoxide and subsequent twofold dilutions were performed in the 96 well microplates to achieve concentrations ranged from 200 to $0.1 \mu \mathrm{g} / \mathrm{mL}$. These concentrations were tested for their activity with estimation of the inhibition \% as described before. The lowest drug concentration causing inhibition of $M$. tuberculosis was considered as the MIC. 


\section{Molecular docking}

The molecular model of the new thiourea derivatives was done using MOE software suite 10.2008. Following geometry optimization, a systematic conformational search was carried out to RMS gradient of $0.05 \AA$ with energy minimization of the resultant conformations employing the ConfSearch module implemented in MOE. All molecular mechanics computations were performed with the Merck Force Field (MMFF94s). The crystallographic structure of $M$. tuberculosis enoyl reductase InhA in complex with $N-\{1-[(2-$ chloro-6-fluorophenyl) methyl]-1H-pyrazol-3-yl]-5-[(1S)-1-(3-methyl-1H-pyrazol-1-yl)ethyl]-1,3,4-thiadiazol-2-amine (GSK 625) was obtained from the Protein Data Bank (PDB ID: 5JFO). Water molecules were ignored and hydrogen atoms were added to the enzyme and partial charges were calculated. Validation followed by docking of the compounds into the active site were carried out, after removing the co-crystallized ligand. The target protein was kept rigid, while the ligands adopt 50 separate docking simulations using default parameters. The conformations were chosen based on their S score, and appropriate fitting with the relevant amino acids in the binding pocket.

\section{Conclusion}

A new series of $N$-(2,6-dimethoxypyrimidin-4-yl)-4-(3(aryl)thioureido) benzenesulfonamides $\mathbf{3 a - t}$ was synthesized. The target compounds were designed and synthesized as potential antitubercular agents. Compounds $3 \mathbf{i}$ and $3 \mathbf{s}$ were found to be the most potent in this study, the reference drug used in this study was isoniazid. Compound $3 \mathbf{i}(\mathrm{MIC}=3.13 \mu \mathrm{g} / \mathrm{mL})$, was the most potent followed by compound $3 \mathrm{~s}(\mathrm{MIC}=6.25 \mu \mathrm{g} / \mathrm{mL})$. Also, the docking study showed that all the docked compounds exhibited similar binding interaction as those previously reported by the co-crystallized ligand when docked into the active site of $M$. tuberculosis enoyl reductase InhA.

\section{Authors' contributions}

MMG, MSAG, MSA were responsible for the organic synthesis, and characterization experiments. AMS was responsible for the docking study. MMA and MME carried out the antimycobacterial activity. All authors read and approved the final manuscript.

\section{Author details \\ 1 Department of Pharmacognosy, College of Pharmacy, King Saud University, P.O. Box 2457, Riyadh 11451, Saudi Arabia. ${ }^{2}$ Department of Drug Radiation Research, National Center for Radiation Research and Technology, Atomic Energy Authority, Cairo 113701, Egypt. ${ }^{3}$ Department of Chemistry, Faculty of Science, Al-Azhar University, Assiut 71524, Egypt. ${ }^{4}$ The Regional Center for Mycology and Biotechnology, Al-Azhar University, Cairo, Egypt.}

\section{Acknowledgements}

The authors would like to extend their sincere appreciation to the Deanship of Scientific Research at King Saud University for its funding of this research through the Research Group Project no. RGP-302.

\section{Competing interests}

The authors declare that they have no competing interests.

\section{Publisher's Note}

Springer Nature remains neutral with regard to jurisdictional claims in published maps and institutional affiliations.

Received: 17 February 2017 Accepted: 16 May 2017

Published online: 19 May 2017

\section{References}

1. Eswaran S, Adhikari AV, Pal NK, Chowdhury IH (2010) Design and synthesis of some new quinoline-3-carbohydrazone derivatives as potential antimycobacterial agents. Bioorg Med Chem Lett 20(3):1040-1044

2. Gemma S, Savini L, Altarelli M, Tripaldi P, Chiasserini L, Coccone SS et al (2009) Development of antitubercular compounds based on a 4-quinolylhydrazone scaffold. Further structure-activity relationship studies. Bioorg Med Chem 17(16):6063-6072

3. Shiradkar M, Kumar GVS, Dasari V, Tatikonda S, Akula KC, Shah R (2007) Clubbed triazoles: a novel approach to antitubercular drugs. Eur J Med Chem 42(6):807-816

4. Zumla A, Nahid P, Cole ST (2013) Advances in the development of new tuberculosis drugs and treatment regimens. Nat Rev Drug Discov 12(5):388-404

5. WHO (2015) Global tuberculosis report 2015. World Health Organization, Geneva

6. Mitnick CD, McGee B, Peloquin CA (2009) Tuberculosis pharmacotherapy: strategies to optimize patient care. Expert Opin Pharmacother 10(3):381-401

7. Stark H, Purand K, Ligneau X, Rouleau A, Arrang J-M, Garbarg M et al (1996) Novel carbamates as potent histamine $\mathrm{H} 3$ receptor antagonists with high in vitro and oral in vivo activity, $\perp$. J Med Chem 39(5):1157-1163

8. Shaw JW, Grayson DH, Rozas I (2015) Synthesis of guanidines and some of their biological applications. Top Heterocycl Chem 50:1-51

9. Shutalev AD (1999) Synthesis and reactivity of 5-functionalized 4-hydroxyhexahydropyrimidine-2-thiones/ones. In: Proceedings of ECSOC-3, the third international electronic conference on synthetic organic chemistry, pp $1-30$

10. Milosavljević MM, Vukićević IM, Drmanić SZ̆, Nikolić JB, Marinković AD, Krstić SS et al (2016) Simple one-pot synthesis of thioureas from amine, carbon disulfide and oxidants in water. J Serb Chem Soc 81(3):219-231

11. Dobrikov GM, Valcheva V, Nikolova Y, Ugrinova I, Pasheva E, Dimitrov V (2013) Efficient synthesis of new (R)-2-amino-1-butanol derived ureas, thioureas and acylthioureas and in vitro evaluation of their antimycobacterial activity. Eur J Med Chem 63:468-473

12. Van Poecke S, Munier-Lehmann $H$, Helynck $O$, Froeyen M, Van Calenbergh $S$ (2011) Synthesis and inhibitory activity of thymidine analogues targeting Mycobacterium tuberculosis thymidine monophosphate kinase. Bioorg Med Chem 19(24):7603-7611

13. Küçükgüzel I, Küçükgüzel SG, Rollas S, Kiraz M (2001) Some 3-thioxo/ alkylthio-1, 2, 4-triazoles with a substituted thiourea moiety as possible antimycobacterials. Bioorg Med Chem Lett 11(13):1703-1707

14. Küçükgüzel I, Tatar E, Küçükgüzel ŞG, Rollas S, De Clercq E (2008) Synthesis of some novel thiourea derivatives obtained from 5-[(4-aminophenoxy) methyl]-4-alkyl/aryl-2, 4-dihydro-3H-1, 2, 4-triazole-3-thiones and evaluation as antiviral/anti-HIV and anti-tuberculosis agents. Eur J Med Chem 43(2):381-392

15. Sriram D, Yogeeswari P, Madhu K (2006) Synthesis and in vitro antitubercular activity of some 1-[(4-sub) phenyl]-3-(4-\{1-[(pyridine-4carbonyl) hydrazono] ethyl\} phenyl) thiourea. Bioorg Med Chem Lett 16(4):876-878

16. Sriram D, Yogeeswari P, Dinakaran M, Thirumurugan R (2007) Antimycobacterial activity of novel 1-(5-cyclobutyl-1, 3-oxazol-2-yl)-3-(sub) phenyl/pyridylthiourea compounds endowed with high activity toward 
multidrug-resistant Mycobacterium tuberculosis. J Antimicrob Chemother 59(6):1194-1196

17. Bhowruth V, Brown AK, Reynolds RC, Coxon GD, Mackay SP, Minnikin DE et al (2006) Symmetrical and unsymmetrical analogues of isoxyl; active agents against Mycobacterium tuberculosis. Bioorg Med Chem Lett 16(18):4743-4747

18. Burger A, Hansch C, Sammes P, Taylor J (1990) Comprehensive medicinal chemistry. Pergamon Press, Oxford

19. Tatar E, Karakuş S, Küçükgüzel ŞG, Öktem Okullu S, Ünübol N, Kocagöz Tet al (2016) Design, synthesis, and molecular docking studies of a conjugated thiadiazole-thiourea scaffold as antituberculosis agents. Biol Pharm Bull 39(4):502-515

20. El-Gaby M, Ismail Z, Abdel-Gawad S, Aly H, Ghorab M (2009) Synthesis of thiazolidine and thiophene derivatives for evaluation as anticancer agents. Phosphorus Sulfur Silicon Relat Elem 184(10):2645-2654

21. El-Gaby MS, Micky JA, Taha NM, El-Sharief S, Marwa A (2002) Antimicrobial activity of some novel thiourea, hydrazine, fused pyrimidine and 2-(4-substituted) anilinobenzoazole derivatives containing sulfonamido moieties. J Chin Chem Soc 49(3):407-414

22. Ghorab MM, Ragab FA, Heiba HI, Soliman AM (2016) Design and synthesis of some novel 4-Chloro-N-(4-(1-(2-(2-cyanoacetyl) hydrazono) ethyl) phenyl) benzenesulfonamide derivatives as anticancer and radiosensitizing agents. Eur J Med Chem 117:8-18

23. El Ella DAA, Ghorab MM, Heiba HI, Soliman AM (2012) Synthesis of some new thiazolopyrane and thiazolopyranopyrimidine derivatives bearing a sulfonamide moiety for evaluation as anticancer and radiosensitizing agents. Med Chem Res 21(9):2395-2407

24. Ghorab MM, Alsaid MS, El-Gaby MS, Safwat NA, Elaasser MM, Soliman AM (2016) Biological evaluation of some new N-(2, 6-dimethoxypyrimidinyl) thioureido benzenesulfonamide derivatives as potential antimicrobial and anticancer agents. Eur J Med Chem 124:299-310

25. Chollet A, Mori G, Menendez C, Rodriguez F, Fabing I, Pasca MR et al (2015) Design, synthesis and evaluation of new GEQ derivatives as inhibitors of InhA enzyme and Mycobacterium tuberculosis growth. Eur J Med Chem 101:218-235

26. Sharma S (1989) Isothiocyanates in heterocyclic synthesis. Sulfur Rep 8(5):327-454

27. Ahmed EM, Taha NM, El-Gawad SMA, Nady NMS (2011) Novel thiourea, quinazoline, thiazolidine, thieno[2,3-d]-pyrimidine, 4-thiazolidinone, pyrrole, pyrrolo[2,3-d]pyrimidine derivatives containing sulfamoyl moiety. Der Chem Sin 2(4):197-210

28. Christensen H, Garton NJ, Horobin RW, Minnikin DE, Barer MR (1999) Lipid domains of mycobacteria studied with fluorescent molecular probes. Mol Microbiol 31(5):1561-1572

29. Maccari R, Ottana R, Vigorita MG (2005) In vitro advanced antimycobacterial screening of isoniazid-related hydrazones, hydrazides and cyanoboranes: part 14. Bioorg Med Chem Lett 15(10):2509-2513

30. Rodrigues MO, Cantos JB, D'Oca CRM, Soares KL, Coelho TS, Piovesan LA et al (2013) Synthesis and antimycobacterial activity of isoniazid derivatives from renewable fatty acids. Bioorg Med Chem 21(22):6910-6914

31. Vistoli G, Pedretti A, Testa B (2008) Assessing drug-likeness-what are we missing? Drug Discov Today 13(7):285-294

32. Vilchèze C, Jacobs J, William R (2007) The mechanism of isoniazid killing: clarity through the scope of genetics. Annu Rev Microbiol 61:35-50

33. Seifert M, Catanzaro D, Catanzaro A, Rodwell TC (2015) Genetic mutations associated with isoniazid resistance in Mycobacterium tuberculosis: a systematic review. PLoS ONE 10(3):e0119628

34. Martínez-Hoyos M, Perez-Herran E, Gulten G, Encinas L, Álvarez-Gómez D, Alvarez E et al (2016) Antitubercular drugs for an old target: GSK693 as a promising InhA direct inhibitor. EBioMedicine 8:291-301

35. Collins L, Franzblau SG (1997) Microplate alamar blue assay versus BACTEC 460 system for high-throughput screening of compounds against Mycobacterium tuberculosis and Mycobacterium avium. Antimicrob Agents Chemother 41(5):1004-1009

\section{Submit your manuscript to a SpringerOpen ${ }^{\circ}$ journal and benefit from:}

- Convenient online submission

- Rigorous peer review

- Immediate publication on acceptance

- Open access: articles freely available online

- High visibility within the field

- Retaining the copyright to your article

Submit your next manuscript at $\boldsymbol{\nabla}$ springeropen.com 